\title{
Wireless Biomedical Sensor Networks: The Technology
}

\author{
Eduardo Freitas, Amândio Azevedo \\ UMa - University of Madeira \\ Caminho da Penteada 9020-105, Funchal, Portugal \\ eduardo.pereira.freitas@gmail.com; jara@uma.pt
}

\begin{abstract}
The increase in research in the area of wireless sensor networks (WSN) has brought a whole new meaning to medical devices. This is mainly due to advances in microcontroller technologies. The WSN are cited as one of the major technologies of this century and hence it assumes importance in areas such as health, psychology, fire prevention, security and even the military. The great advantage of this technology is the ability to track, monitor, study, understand and act on a particular phenomenon or event. The primary purpose of a wireless health system is reliable data transfer with minimum delay. This work is a synthesis of vast research done as Wireless Biomedical Sensor Networks (WBSN), including experimental and non-experimental investigations as well as data from the theoretical and empirical literature which incorporates a wide range of purposes: definition of concepts, review theories and evidence analysis of methodological problems, seeking to generate a consistent and understandable overview of WBSN. Such systems are already being marketed, some are still under investigation. It is also the aim of this study to identify the characteristics of a WSN applied to health.
\end{abstract}

Keywords: Wireless Biomedical Sensor Networks, Biosensors, Healthcare telemetry system

\section{Introduction}

Overall, the elderly population are growing and generally getting older. Life expectancy continues to increase with the new advances in healthcare. Today, over 850 million people worldwide suffer from chronic diseases and spend up to $85 \%$ of their savings in healthcare plans. It is always better to prevent a disease than to treat it, so that individual monitoring is required as a periodic activity. The growing population in developed countries leads governments to increase their healthcare budgets, and also presents new challenges to health systems, especially with older people living either on their own or in old age homes. [1] [2]

Due to the increase of research in the area of wireless sensor networks (WSN), these have brought new opportunity in medical devices. The WSN are seen as one of the most important advances in technology of this century and hence its importance in areas as diverse as health, psychology, fire prevention, security and even in the military. The advantage of this technology is the ability to track, monitor, study, understand and act on a particular phenomenon or events. [3]

Traditionally, healthcare monitoring is performed on a periodic check basis where patients are constantly updated on their symptoms; the physician checks and makes a"diagnosis, then when possible monitors the patient's progress during treatment. In most cases, health monitoring is done by wireless network infrastructures. But the coverage of these network infrastructures has limitations from bandwidth. These limitations in continued health surveillance services, it is not always possible to send emergency signals from patients to healthcare workers. With WSN, patients can get continuous health monitoring using wireless ad hoc networks which can transmit vital signs over shorts distances. In most systems, the health data of multiple patients may be resent using the wireless multi-jump routing scheme for a base station. [4]

Already telemedicine systems can establish news protocols such as IEEE standards 802.11, 802.15 and 802.16, which are characterized by the distribution networks for medical information providing life-saving services. [5]

Wireless sensors can be placed on patients in a hospital or homecare setting to gather physiological signals. The correct WSN design depends on accurate traffic models, the selection of the correct model is essential for the correct management of network traffic, network congestion, interference between nodes and the energy expended by each node. Currently, there are no traffic models that represent medical WSN applications. [6] 


\section{Node Sensor Wireless Sensor Network}

Over the last years there has been a significant increase in the number of various wearable health monitoring devices, to measure physiological signals. However, these systems are still limited and they have important restrictions. For example, Holter monitors are used only to collect data, and the data processing and analysis are performed offline, because that limitation, the device became impractical for continued monitoring and early detection of medical disorders. Systems with multiple sensors for physical rehabilitation normally have wires between the sensors and the monitoring system that are a restriction to a patient's activity and level of comfort. [7]

The most recent technology advances in integration and miniaturization of physical sensors, embedded microcontrollers and radio interfaces on a single chip; wireless networking; and micro-fabrication have enabled a new generation of wireless sensor networks suitable for many applications which arouses great interest in health monitoring and rehabilitation. The physiological sensors that monitor vital signs, environmental sensors (temperature, humidity, and light), and a location sensor can all be integrated into a Wearable Wireless Body/Personal Area Network (WWBAN) or WBSN. [7] The Table 1 shows the differences between the WSN and WWBAN.

Table 1: Different challenges faced by WSN and WWBAN [5] [8] [9] [10] [11] [12] [15] [36].

\begin{tabular}{|c|c|c|}
\hline & WSN & WWBAN \\
\hline Scale & Wide are coverage $(\mathrm{m} / \mathrm{km})$ & Limited by the human body $(\mathrm{m} / \mathrm{cm})$ \\
\hline Node number & Huge number of nodes for coverage & Limited number of pervasive nodes \\
\hline Function & Multiple sensors, each perform dedicated tasks & Single sensors, each perform multiple tasks \\
\hline Accuracy & Compensated by the redundancy & Accurate measurements are required by each node \\
\hline Size & Small size preferable but not a major limitation & Pervasive monitoring and need for miniaturization \\
\hline Dynamics & $\begin{array}{l}\text { Exposed to extremes in weather, noise, and } \\
\text { asynchrony }\end{array}$ & Exposed to more predictable environment \\
\hline Event detection & $\begin{array}{l}\text { Early adverse event detection desirable; failure } \\
\text { often reversible }\end{array}$ & $\begin{array}{l}\text { Early adverse events detection vital; human tissue } \\
\text { failure irreversible }\end{array}$ \\
\hline Variability & $\begin{array}{l}\text { Much more likely to have a fixed or static } \\
\text { structure }\end{array}$ & $\begin{array}{l}\text { Biological variation and complexity means a more } \\
\text { variable structure }\end{array}$ \\
\hline Data Protection & $\begin{array}{l}\text { Lower level wireless data transfer security } \\
\text { required }\end{array}$ & $\begin{array}{l}\text { High level wireless data transfer security required } \\
\text { to protect patient information }\end{array}$ \\
\hline Power supply & $\begin{array}{l}\text { Accessible and likely to be changed more easily } \\
\text { and frequently }\end{array}$ & $\begin{array}{l}\text { Inaccessible and difficult to replace in implantable } \\
\text { setting }\end{array}$ \\
\hline $\begin{array}{l}\text { Energy } \\
\text { Scavenging }\end{array}$ & Solar, and wind power & Motion (vibration) and thermal (body heat) \\
\hline Failure & Nodes often disposable & Difficult replacement of implanted nodes \\
\hline Access & $\begin{array}{l}\text { Sensors more easily replaceable or even } \\
\text { disposable }\end{array}$ & $\begin{array}{l}\text { Implantable sensor replacement difficult and } \\
\text { requires biodegradability }\end{array}$ \\
\hline Biocompatibility & Not a consideration in most applications & $\begin{array}{l}\text { A must for implantable and some external sensors. } \\
\text { A chemical reaction with human tissue that may } \\
\text { have the biosensor. Increase cost }\end{array}$ \\
\hline $\begin{array}{l}\text { Context } \\
\text { Awareness }\end{array}$ & Not important with static sensors & $\begin{array}{l}\text { Very important, because physiology is sensitive to } \\
\text { context change }\end{array}$ \\
\hline $\begin{array}{l}\text { Wireless } \\
\text { technology }\end{array}$ & Blueetooth, Zigbee, GPRS, Wireless LAN, RF & Low power wireless required \\
\hline Data transfer & $\begin{array}{l}\text { Loss of data during wireless is compensated by } \\
\text { number of sensors used }\end{array}$ & $\begin{array}{l}\text { Loss of data more significant, and may require } \\
\text { additional measures to ensure QoS and real-time } \\
\text { data interrogation capabilities }\end{array}$ \\
\hline
\end{tabular}

There are standards for personal health devices the interoperability of such as scales, blood pressure monitors and blood glucose monitors. This family of standards is based around a framework defined the IEEE 11073-20601-2008 standard 
and its amendment, IEEE 11073- 20601a-2010. The Personal, Home and Hospital Care (PHHC) profile developed by the ZigBee Alliance relies on the on-going work being developed by IEEE 11073 to allow the interoperability with medical devices. [13]

\section{Wireless Biomedical Sensor Biomedical}

Information technology companies are aware of the increasing interest and a demand for this technology and they are designing m-health solutions such as eWatch or LifeShirt. As a result, wireless sensor network application to Healthcare presents effective commercial solutions for the general public and for not only hospital research facilities. [2]

\subsection{Important measures to Medical Applications}

Nowadays the early detection is important, diseases or monitoring patient's is vital for human survival in extreme situations.

There are several parameters/diseases that can be measured or detected, such as:

- Cancer detection: Today, there is no conclusive evidence on how to prevent cancer, but it's detection is possible and it is crucial. Studies have shown that cancer cells exude nitric oxide, which affects the blood in the area surrounding a tumor. [9]

- Glucose Level Monitoring: Can be measure by a biomedical sensor, which will be monitoring the glucose level. This method can provide a more consistent, accurate, and less invasive. [9]

- Asthma: The sensors can detect the allergens in the air and report the status continuously to the physician and/or to the patient. The server also collects information from the network of national monitoring stations of air quality. [9]

- Cardiovascular diseases and heart rate: sensors which are placed discretely allowing the physicians to receive the patient's vital information and so prepare treatment while monitoring their patient's health.

- Alzheimer, depression and the elderly: In these situations it is possible to detect abnormal situations, such as falls, and it can alert neighbors, family or the nearest hospital. It can work with accelerometers to detect these movements and may use the ZigBee protocol or via GSM to provide real-time information. It can also use RFID readers to control the inputs and outputs of the patient; sound sensors can detect motion and request assistance and the light to check opening of refrigerator to monitor how often patient get food. [9] [14]

- Artificial retina: 100 micro sensors are used which are implanted inside the eye, these sensors produce electrical signals, then the underlying tissue converts the signals into a chemical response, reproducing the behavior of normal retinal light stimulation. [9] [12]

These are some of the parameters that can be measured in the medical applications, however all of these measurements can be applied in various situations. It is one of the areas still under development to better the quality of life.

\subsection{The WSN access to information of interest in Medical Applications}

Advances in technology have allowed the integration and miniaturization of sensors, embedded microcontrollers and radio interfaces in a single microcontroller; WSN and microfabrication have allowed a new generation of WSN suitable for many applications. One of the most exciting and important areas of application are in health supervision. WBSN refers to the wireless body area network (WBAN) and it uses a wireless architecture that consists of a number of body sensor units (BSUs) combined with a single body central unit (BCU). This network is made up of wearable computing devices which are in development. The WBAN is an on-body sensor having little power to include wireless devices for easy isolated control. In real time, the situation of several patients is being monitored constantly in this type of architecture. So it can monitor physiological parameters. However, this type of application has faced several challenges when designed. For example, communications between the sensors need to be reliable and free of interference and it should also provide flexibility to the users. The growth of WBAN must include the advance of diagnostic tools of the medical monitoring system. [15]

A variety of physiological sensors that monitor vital signs, environmental sensors (temperature, humidity and light), and a location sensor may be integrated in a WWBAN. The WWBAN is constituted by inexpensive, lightweight and miniature sensors, and it may allow a long-term, discrete and ambulatory health monitoring with instantaneous feedback to the user about the current state of health in real-time and the user's medical records. Such a system can be used to supervise the rehabilitation by computers in multiple conditions, and even early detection of medical conditions. For example, intelligent heart monitors can alert patients about their medical conditions or provide information to a specialized service in 
the event of catastrophic events. When integrated in a broader telemedicine system with the patient's medical records, WWBAN shows a revolution in medical research by sending all collected data. The large amount of collected physiological data will allow quantitative analysis of various conditions and patterns. [7]

Recent advances in miniaturization of intelligent biosensors opened new opportunities for the continuous monitoring of patients. [9] Unobtrusive tiny wearable sensors will collect large amounts of data automatically, reducing the cost and inconvenience of regular visits to the physician. [9]

\subsection{The comparison of technologies used in WSN in Medical Applications}

In this area the WSN has a wide application and can be used in almost all applications, and all technologies used in WSN can be applied to health, including sensors and positioning systems such as GPS. [5] For example, the wireless data communication is a bidirectional radio frequency communication with ad-hoc routing, which allows each patient's node to send the data to a base station, even if they are not within its direct radio range. [16] As shown in the following figure:

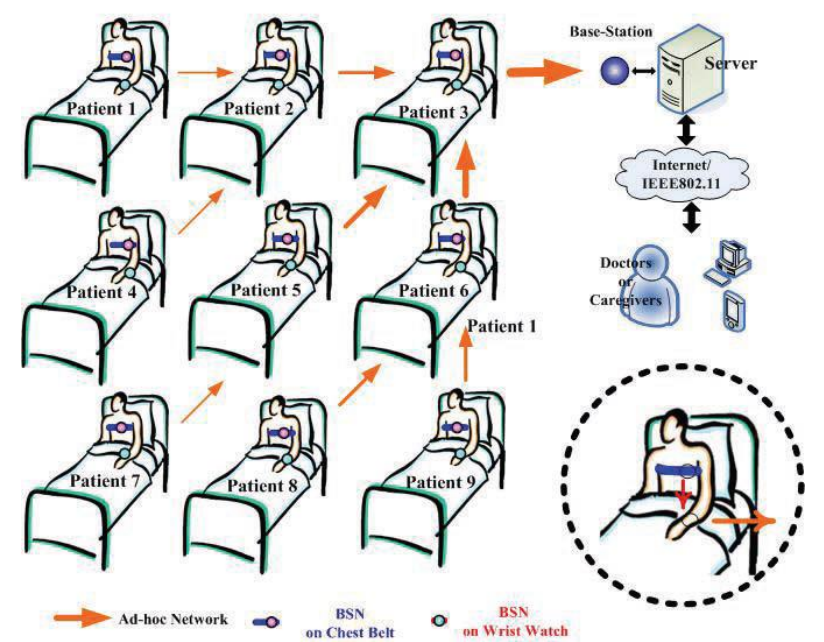

Fig. 1: Architecture example of a healthcare system based on a WSN [16].

Aminian and his team designed a prototype of a ubiquitous health system for hospitals, which is the concept of ubiquitous, placing wireless sensors unobtrusively in a person's body to form a wireless network that can communicate the state of health of the patient with the base station connected to the PC monitor. However, the relay nodes in the middle do not require use of a high frequency band and can transmit the data over a short range frequency module (RFM). But it is important to note that, there are medical bands such as MICS (Medical Implant Communication Service), 10 meters of 402-405 MHz frequency band coverage that can be used for body area network applications because of their low power transmissions. [4] Tolentino and his team presented an architecture of a health system ubiquitous for continuous monitoring of patients in their natural physiological conditions or elderly patients with chronic diseases, the biggest difference it is designed to monitor the elderly who live in remote areas or in small nursing home without enough health technical support, instead of monitoring patients in a large hospital environment. This WSN ad hoc is used integrated with existing medical practices and technologies in real time remote monitoring to provide medication and a patient status monitoring system assisted by incorporated the wireless sensor. The system transfers the data wirelessly to a base station connected to a server on an ad hoc network using IEEE 802.15.4 or LR-WPANs. [18] Sheltami and his colleagues developed a telemetry project with the definition of alerts priorities depending on the emergency. In this WSN, profiles of patients are updated with the information processed in the central database. The central computer is responsible for sending e-mails and/or messages in case of emergencies. [19] [20] Mbakop and his team developed a monitoring of patients in real-time systems, which is made up by a system of two nodes where vital signs are collected and transmitted wirelessly to a base station and then the data can be stored and presented on a continuous base station. The great innovation in this design is the correct operation, but the biggest challenge is its' actual use in the future of the movements of the patient to produce energy for sensors [21]

The home monitoring is one of the examples being developed with the WSN, not only to detect environmental parameters that can affect patients but also to help in memory and other healthcare and security services. [9] Gaddam and 
his team developed and implemented a SMART, it is based on Selective Activity Monitoring (SAM) and it integrates various intelligent sensors which communicate via the standard radio protocols. The system depends on a number of selected set of wireless intelligent sensors and a controller. This controller receives and processes inputs from the sensors. The selected sensors are installed as monitor in televisions, reading lamps and beds, in different locations in a house. [17]

Walker and his team showed the need to create a single platform, but robust enough to support a number of complex applications. The fundamental technical contributions of the proposal and the improvements platform include: 1) the application of JADE (Java Agent-based Development framework) for WSN environment, 2) the abstraction of sensors and types of events, 3) a user interface for application development, and 4) Architecture used to monitor the application states. They monitored patient's behaviour and health, when using a health professional system or a relative in question would be able to plan the application to monitor the patient's routine, and define actions based on detection of significant events with sensors in the elderly's house. [14]

The WearIT@work project was established by the European Commission as an integrated project to investigate "Wearable Computing" as a technology dealing with integrated computer systems in clothing (wearIT@work). One possible application of this project is the rapid availability of patient's medical information at any time; this could mean an interesting reduction in costs in medical examinations, also the power to perform medical evaluations on a daily basis and according to the circumstances of patients and in extreme cases, it could save patient's lives. [5]

Alarm-Net presents preventive healthcare an adaptable solution for continuous exams over wireless sensors for intelligent medical care, creating a historical record and preserving the privacy of the patient. These sensors can detect small changes but key signals that humans cannot see and alert to like heart rate, oxygen levels in the blood and the circadian rhythm which may indicate changes in health requirements. [15] Alarm-Net accomplishes these goals through the unification and aggregation of heterogeneous devices in a common architecture. [22]

CodeBlue (developed by Harvard University) is a wireless infrastructure intended to provide a common protocol and software structure in a disaster scenario response, allowing monitoring and wireless monitoring of patients and rescuers. The CodeBlue is a self-organized platform that is easy to connect because of its ad hoc architecture and it integrates different nodes of wireless device sensors. The system integrates low-power wireless sensors, and it offers services for the establishment of credentials, handoff, location tracking, and network's filtering and aggregating of produced data by the sensor. The simple interface allows emergency medical technicians to request data from a group of patients. The CodeBlue is designed to pass through a wide range of density networks and operates in a range of wireless devices, from the limited resources to the PDA and more powerful PCs. The CodeBlue has several types of sensors (oximetry, ECG and motion sensor) and it is used together with the ZigBee trading platforms, Mica2, MICAz and Telos. Researchers consider that such platforms have a good response in research settings but have many failings in actual scenarios due to the dimensions of the modules and batteries; a support platform has also been developed for lighter sensors to be used in accident monitoring modules in a non-invasive way. [9] [15] [23] [24]

The MEDiSN is similar to the CodeBlue, but it is using a different architecture. [25] The value includes a number of physiological monitors (PMs) - nodes on which the physiological patient data is collected. Each node has sensors with a configurable frequency, physiological encrypted data, and transmits them to a network of relay points (RPs). The RPs transmit the data to the PMs gateway and the gateway management data to one or more PMs. To support this traffic in both directions, the RPs auto configures in a routing tree with PMs in its branches. While RPs are stationary, PMs can be mobile and periodically select the best RP to transmit the data. [26]

NetCare was a Portuguese project developed by Inovamais in partnership with Agilus I+D, the Engineer at the Faculty of Oporto University, São Sebastião Hospital and Speculum. This project was developed a monitoring platform where healthcare services such as emergency and ambulances were interconnected and physicians can observe the situation of many patients. This information was available on a web application. [27]

AID-N handles mass incidents like accidents. Instead of implementing APs (access policy) on the wall, wireless repeaters are positioned for an emergency course set. When APs are flashing green lights, the medical team is aware of a precise emergency. [15] The system consists of the four blocks MiTags (MiTags are modules and extensible wireless sensor's platforms. These modules support two types of communication: short range, so as to be able to create a WBAN and long range, so it can connect to support mesh. The level sensor, the platform supports several types: blood pressure, temperature, $\mathrm{SpO}_{2}$ and others.) routers, devices to access to MiTags and a central server. [23] 
The company eHit is a Finnish company that provides services and technologies related to healthcare at a distance. It is one of the technologies of the Health Gateway, a platform which connects to a mobile device and a server. The mobile device has as function to collect data from the sensors and send it to the server, and the server's aim is to receive data and forward it to be processed. However the eHit company has more products in its portfolio, including the Pacient Care Device Gateway, which is similar to the Health Gateway, but allows monitoring in the hospital without a mobile device, using the LAN connection; and also eLink is a module connecting the sensors which it has not a Bluetooth connection, and so it is connected to the mobile device. [23]

The HipGuard is a system and it is developed for patients who are recovering from hip replacement surgery. This system monitors the patient's leg, hip's position and rotation with wireless sensors. The alarm signals are sent to the patient's wrist unit. If his hip or leg positions or rotations are false, and therefore the HipGuard system provides useful information in real time to the patient rehabilitation process. [12]

An area that is still challenging is the detection of epileptic seizures and convulsions; however, researchers at the Medical Center of the University of Chicago developed a device called a Mobi to detect abnormal brain activity, broadcasts signals of abnormal electrical activity warning the receiver. [12]

Also, the artificial retina, which is intelligent sensors integrated microsystems (SSIM) retinal prosthesis of the chips which are formed of 100 microsensors, constructed and implanted a human eye. Wireless communication is used to meet the need of control, image identification and validation, as these sensors produce electrical signals, the underlying tissue converts the signals into a chemical response, and thus simulates the retinal behavior with stimulation by light. [9] [12]

The Psychology Department of the Institute of Technology Georgia more specifically, the Georgia Tech Sonification Lab also identified a similar problem in vision, They created the SWAN project, a practical and portable device which features navigation software for people without vision or even in places where vision is limited, and it emphasized the need to avoid obstacles or for environmental characteristics quickly. [5]

\section{Applications in healthcare}

For the detection of vital signs and various physiological data various technologies have been developed: Vital Signs Monitoring System, LifeShirt, Fireline, UbiMon, Satire Project, SMART, HealthGear, MobiHealth/Mobicare, CareNet, Jacket Vital, BikeNet, eWatch, AMON project, MagIC System, Smart Vest, Secure Mobile Computing using Biotelemetrics and SenseWear Armband. [9] [12] [15] [22] [23] [27] [28] [29] [30] [31] [33]

Another technology that has been used is the endoscopic capsule. This method for recording images of the digestive tract used in medicine, allows a small chamber, the size of a tablet which allows you to view images of the inside of the gastrointestinal tract and it is possible to check areas where using the traditional forms of endoscopy is not possible. [34]

One of the areas which show more interest among researchers is the area of babies, not only for being a lucrative area, but also as a concern and a need to help prevent the sudden infant death syndrome (SIDS). From that the invention of SleepSafe monitors the child's sleeping position and alert parents. [35] Another solution is the Baby Glove, this project is to protect premature infants, as they are subject to various health risks. [35] [35] [29]

The use of WSN in biomedical applications facilitates the development of wearable systems, making them lighter and less obtrusive for the elimination of the cables. The wearable systems are computer systems integrated into consumer items used as garments. Therefore, a need exists to respond to various aspects:

- the insertion mechanisms and policies to reduce energy consumption and increase the durability of the monitoring and detection of the data collected by the sensors;

- development of a protocol to provide biomedical signals in a database on the Internet, where data would be accessed by physicians. Thus, there would be several advantages such as: a quicker realization of exams, and providing reliable data, cost reduction and convenience in carrying out the examinations;

- in the study and implementation of mechanisms for authentication and information security. [37]

In terms of protocols, several issues such as robustness, security and communication without interference are key requirements. It is believed that the role of wireless sensor networks in medicine can be expanded further. In the near future, smart spaces incorporated with wireless sensor networks can detect environmental conditions and take preventive measures based on that knowledge. The system can achieve ubiquity, where each one will have a computational module capable of interacting with the system of the smart area and avoid health problems. 


\section{Conclusion}

After reviewing several articles and research, that has been conducted, we may conclude that: there is still a long way to go in the area of wireless sensor network. Existing medical applications based on sensor networks are in the first-line potential research for use in the future of WSNs and their medical device looks extremely promising. Security issues are a significant area, and there are still a number of large challenges to overcome.

The future should include specialized medical technology with WSN, where with the existing infrastructure enhances the collection of data in real time, in which the medical care at home and smart homes will be improve. Also the constant collection of clinical data of patients will reduce the costs of tests and regular visits to the physicians. Another important point in the future will is the relationship between bioscience, biotechnology and nanoscience (nanotechnology) in the development of sensors.

\section{References}

[1] A. Minaie, A. Sanati-Mehrizy, P. Sanati-Mehrizy, and R. Sanati-Mehrizy, "Application ofWireless Sensor Networks in Health Care System," em 120th ASEE Annual Conference \& Exposition, 2013.

[2] P. Neves, J. Fonseca, and J. Rodrigues, "Simulation Tools for Wireless Sensor Networks in Medicine: A comparative study"

[3] T. Camilo, A. Rodrigues, J. S. Silva, and F. Boavida, "Redes de Sensores Sem Fios, considerações sobre a sua instalação em ambiente real," Coimbra, pp. 89-98.

[4] M. Aminian and H. Naji, "A Hospital Healthcare Monitoring system using wireless," Health \& Medical Informatics, vol. 4, 2013.

[5] C. Lozano, C. Tellez, and O. Rodríguez, "Biosignal Monitoring Using Wireless Sensor Networks," em Biomedical Engineering, Trends in Electronics, Communications and Software, 2011.

[6] G. G. Messier and I. G. Finvers, "Traffic Models for Medical Wireless Sensor Networks," IEEE Communications Letters, vol. 11, pp. 13-15, 2007.

[7] A. Milenkovic, C. Otto, and E. Jovanov, "Wireless Sensor Networks for Personal Health Monitoring: Issues and an Implementation," Huntsville.

[8] P. Honeine, F. Mourad, M. Kallas, H. Snoussi, H. Amoud, and C. Francis, "Wireless sensor networks in biomedical: Body Area networks."

[9] P. Neves, M. Stachyra, and J. Rodrigues, "Application of Wireless Sensor Networks to Healthcare Promotion,” 2008.

[10] M. Barua, M. Alam, and L. X. Xiaohui, "Secure and quality of service assurance scheduling schem for WBAN with application to eHealth," em Wireless Communications and Networking Conference, 2011.

[11] A. Darwish and A. E. Hassanien, "Wearable and Implantable Wireless Sensor Network Solutions for Healthcare Monitoring," Sensors, pp. 5561-5595, 2011.

[12] N. Fatema and R. Brad, "Security Requirements, Counterattacks and Projects in Healthcare Applications Using WSNs - A Review," International Journal of Computer Networking and Communication (IJCNAC), vol. 2, 2014.

[13] J. Stankovic, Q. Cao, T. Doan, L. Fang, Z. He, R. Kiran, S. Lin, S. Son, R. Stoleru, and A. Wood, "Wireless Sensor Networks for In-Home Healthcare: Potential and Challenges."

[14] P. Khan, M. Hussain, and K. Kwak, "Medical Applications of Wireless Body Area Networks," International Journal of Digital Content Technology and its Applications, vol. 3, 2009.

[15] H. F. López, "Remote Vital Signs Monitoring Based on Wireless Sensor Networks," 2011.

[16] Z. Walker, M. Moh, and T.-S. Moh, "Development Platform for Wireless Sensor Networks with Biomedical Applications," IEEE, pp. 768-772, 2007.

[17] W.-Y. Chung, "Ubiquitous Healthcare System Based on a Wireless Sensor Network," Oulu University Press, Oulu, 2009.

[18] A. Gaddam, C. Mukhopadhyay, and G. S. Gupta, "Elder Care Based on Cognitive Sensor Network," IEEE Sensors Journal, vol. 11, pp. 574-581, 2011.

[19] R. Tolentino, Y. Kim, B. Park, and G. Park, "A Design and Analysis Ubiquitous Healthcare Monitoring System over Wireless Sensor Network," International Journal of Multimedia and Ubiquitous Engineering, vol. 6, pp. 55-69, 2011.

[20] T. Sheltami, A. Mahmoud, and M. Abu-Amara, "An Ad hoc wireless sensor network for Telemedicine applications," The Arabian Journal for Science and Engineering, vol. 32, pp. 131-143, 2006. 
[21] T. Sheltami, A. Mahmoud, and M. Abu-Amara, "An ad hoc wireless sensor network for telemedicine applications," The Arabian Journal for Science and Engineering, vol. 32, pp. 131-143, 2007.

[22] A. Mbakop, A. Lambebo, L. Jayatilleke, and S. Haghani, "Implementation of a Wireless Body Area Network for Healthcare Monitoring," ASEE - American Society for Engineering Education.

[23] J. Stankovic, A. D. Wood, and T. He, "Realistic Applications for Wireless Sensor Networks."

[24] S. Leroux, "Convergência entre dispositivos móveis e redes de sensores sem fios," 2013.

[25] M. Welsh, "Wireless Sensor Networks for Emergency Medical Care," Harvad University, 2004.

[26] J. Ko, C. Lu, M. B. Srivastava, and J. A. Stankovic, "Wireless Sensor Networks for Healthcare," Proceedings of the IEEE, pp. 1947-1960, 2010.

[27] J. Ko, J. H. Lim, R. Musaloiu-e., A. Terzis, G. M. Masson, T. Gao, W. Destler, L. Selavo, and R. P. Dutton, "MEDiSN: Medical Emergency Detection in Sensor Networks," ACM Transactions on Embedded Computing Systems, vol. 10, 2010.

[28] J. P. S. Oliveira, "Desenvolvimento e integração de sensores numa Plataforma para sistemas de Monitorização Pessoais," Porto, 2012.

[29] S. Stankovic, "Medical Applications Based on Wireless Sensor Networks," pp. 19-23.

[30] P. Gonçalves, "Monitorização Remota de Pacientes em Ambulatório," Porto, 2008.

[31] M. Rienzo, F. Rizzo, G. Parati, G. Brambillan, M. Ferratini, P. Castiglioni, P. Meriggi, P. Mazzoleni, and B. Bordoni, "MagIC system," IEEE Engineering in Medicine and Biology Magazine, vol. 28, pp. 35-40, 2009.

[32] P. Pandian, K. Mohanavelu, K. P. Safeer, T. M. Kotresh, D. T. Shakunthala, P. Gopal, and V. C. Padaki, "Smart Vest: wearable multi-parameter remote physiological monitoring system," Medical engineering \& physics, vol. 30, pp. 466477, 2008.

[33] BodyMedia, Inc. (2015, June 20). SenseWear - Learn More. [Online]. Available: http://sensewear.bodymedia.com/SWLearn-More/How-SenseWear-Works.

[34] S. Rajasekaran, P. Kumuran, G. Premnath, and M. Karthik, "Human Health Monitoring using wireless sensors network," International Journal of Application or Innovation in Engineering Management, vol. 2, pp. 323-330, 2013.

[35] C. Baker, K. Armijo, S. Belka, M. Benhabib, V. Bhargave, N. Burkhart, A. Minassians, G. Dervisoglu, L. Gutnik, M. Haick, C. Ho, M. Koplow, J. Mangold, S. Robinson, M. Rosa, M. Schwartz, C. Sims, H. Stoffregen, A. Waterbury, and E. Leland, "Wireless Sensor Networks for Home Health Care," em 21st International Conference on Advanced Information Networking and Applications Workshops, 2007.

[36] E. M. Gutiérrez, A. F. Rocha, T. M. G. Andrade, D. R. França, and H. S. Carvalho, "Redes de Sensores Sem Fios, considerações sobre a sua instalação em ambiente real," Coimbra. 